\title{
Una población de Broussonetia papyrifera (L.) Vent. naturalizada en el interior de la Península Ibérica
}

\author{
Juan Quesada \& Carlos Salazar \\ Dpto. Biología Animal, Biología Vegetal y Ecología. Facultad de Ciencias Experimentales. Universidad de Jaén. E-23071- \\ Jaén (España)
}

\section{Correspondencia}

C. Salazar Mendías

e-mail: csalazar@ujaen.es

Recibido: 6 mayo 2019

Aceptado: 5 marzo 2020

Publicado on-line: 3 septiembre 2020

Editado por: Marta Recio Criado

\section{RESUMEN}

En el presente trabajo se cita una población naturalizada de Broussonetia papyrifera (L.) Vent localizada, por primera vez, en las riberas del rio Tajo (Toledo). Dado el potencial invasor de esta especie se propone la necesidad de su control y vigilancia.

Palabras clave: Castilla-La Mancha, Toledo, Río Tajo, plantas invasoras, vegetación riparia

\begin{abstract}
A naturalized population of Broussonetia papyrifera (L.) Vent. in the interior of the Iberian Peninsula

In the present work, a naturalized population of Broussonetia papyrifera (L.) Vent located, for the first time, in the banks of the Tajo river (Toledo) is mentioned. Given the invasive potential of this species, the need for its control and monitoring is proposed.
\end{abstract}

Key words: Castilla-La Mancha, Toledo, Tagus river, alien plants, riparian vegetation
Broussonetia papyrifera (L.) Vent. es un macrofanerófito de la familia Moraceae originario del este de Asia que ha sido utilizado para fabricar papel desde antiguo. En España es una especie alóctona, que se ha considerado propia de ambientes ruderales y viarios (Sanz-Elorza et al. 2004).

Es una planta caducifolia y dioica de porte arbóreo (1-6 m) con copa redondeada y corteza lisa grisácea. Los pies masculinos tienen amentos péndulos y cilíndricos mientras que los pies femeninos tienen flores en glomérulos tomentosos verdes que se vuelven rosados en la madurez (Guillot Ortiz, 2009).

A pesar de ser originaria del este de Asia, se ha extendido a numerosas partes del planeta. De hecho, actualmente tiene una distribución bastante amplia (Figura 1) y se ha extendido a regiones como Europa, Norteamérica, Sudamérica, Madagascar y Oceanía, así como a numerosas islas del Pacífico (GBIF, 2020).

En la provincia de Toledo se ha detectado una población naturalizada de $B$. papyrifera en la ribera de río Tajo (Santiago de Villarrubia) (Figuras 2, 3 y 4). Se ha depositado un pliego testigo en el Herbario de la Universidad de Granada (GDA). En el presente trabajo se discuten algunos aspectos generales relacionados con la especie, y se realizan valoraciones sobre la población encontrada.

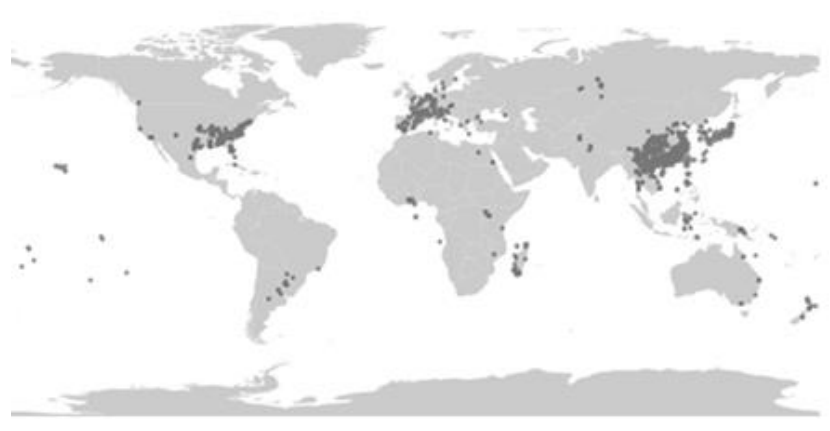

Figura 1. Distribución de Broussonetia papyrifera en el mundo. Fuente Global Biodiversity Information Facility (consultado en junio de 2020).

Figure 1. Worldwide distribution of Broussonetia papyrifera. Source Global Biodiversity Information Facility (accessed June 2020).

Broussonetia papyrifera (L.) Vent. in Tabl. Regn. Vég. 3: 547 (1799)

ESPAÑA. Toledo: Villarrubia de Santiago, Río Tajo, cerca de la intersección con el Arroyo Valdeajuelos. 30T472940/4432123, $522 \mathrm{~m}$., $23 / 06 / 2020$, Planta naturalizada en la ribera del río Tajo. Varios ejemplares en la ribera, J. Quesada (GDA 66631). (Figuras 3 y 4). 

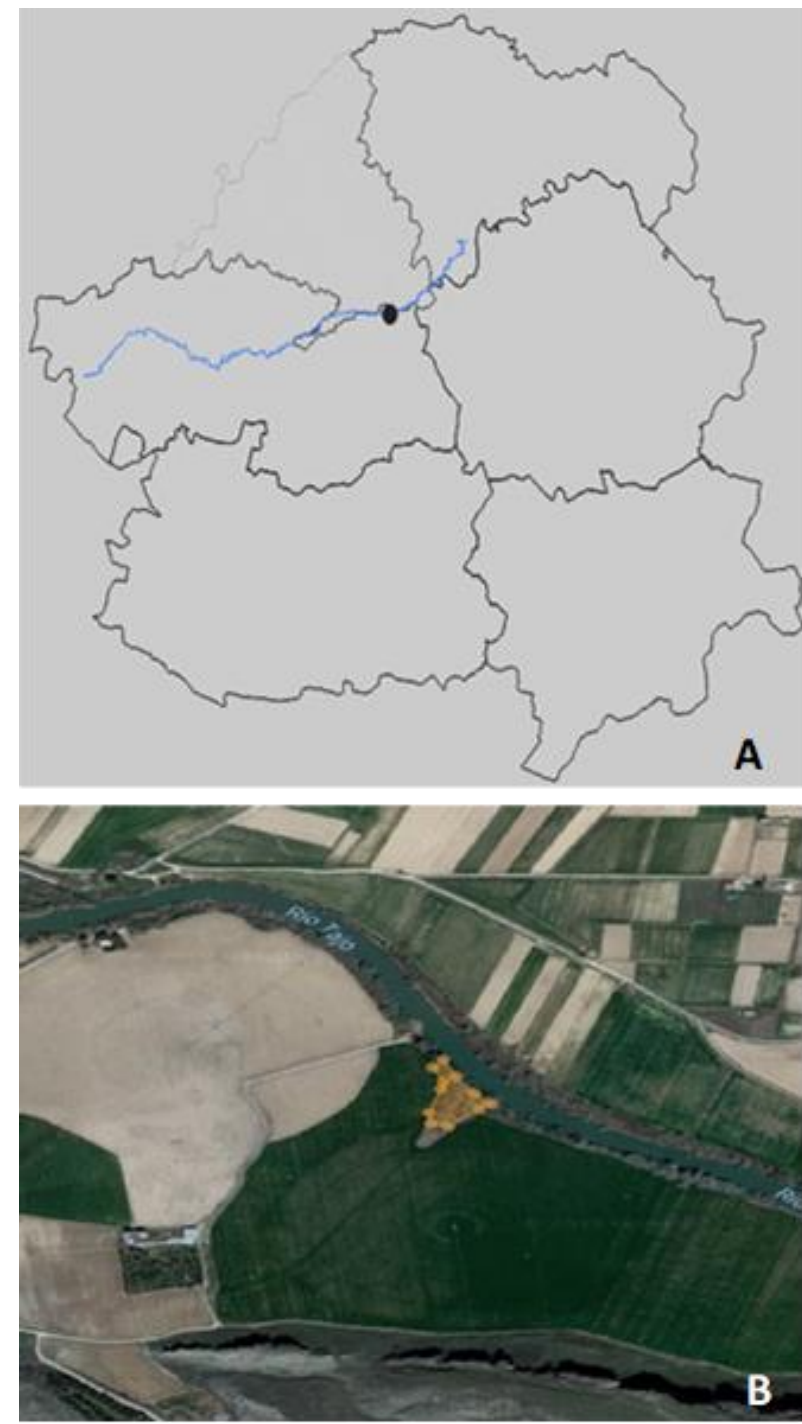

Figura 2. A) Localización de la población de B. papyrifera detectada en el río Tajo en la provincia de Toledo (coordenadas 472940/4432123, ETRS89). B) En naranja se delimita la presencia de esta población.

Figure 2. A) Location of the population of B. papyrifera detected in the Tagus River, in the province of Toledo (coordinates 472940/4432123, ETRS89). B) The presence of this population is delimited in orange.

\section{Distribución y evolución del carácter alóctono en España}

En la Península Ibérica existen numerosas referencias de la presencia de $B$. papyrifera, sobre todo, en España. La mayoría en el Levante (Figura 5), especialmente en Cataluña y, en menor medida, Valencia. En el resto del país su distribución es más irregular y suele aparecer en enclaves dispersos y ligados a zonas urbanas. En Portugal, aunque no se han encontrado referencias en la base de datos GBIF, existen trabajos que denuncian su presencia (De Almeida, 2006).

En España, el carácter alóctono de B. papyrifera ha ido evolucionando, desde ornamental, hasta llegar a naturalizarse. Inicialmente, todo apunta a que se introdujo en jardines. Guillot Ortiz (2009) recoge varias referencias de finales del siglo XIX y principios del $X X$, en las que se la cita en este tipo de zonas. En ese periodo, solo se ha encontrado una referencia que aluda a una posible naturalización (Laguna, 1870), concretamente en Montealegre (provincia de Barcelona), donde se habla de ejemplares con "aspecto de asilvestrados". Casi 100 años después, varios autores coincidieron en que crecía cultivada en jardines, pero que puntualmente podía naturalizarse (Bolós \& Vigo 1990; Castroviejo 1993). En la actualidad, está incluida en la flora alóctona de España (Sanz-Elorza et al. 2004).

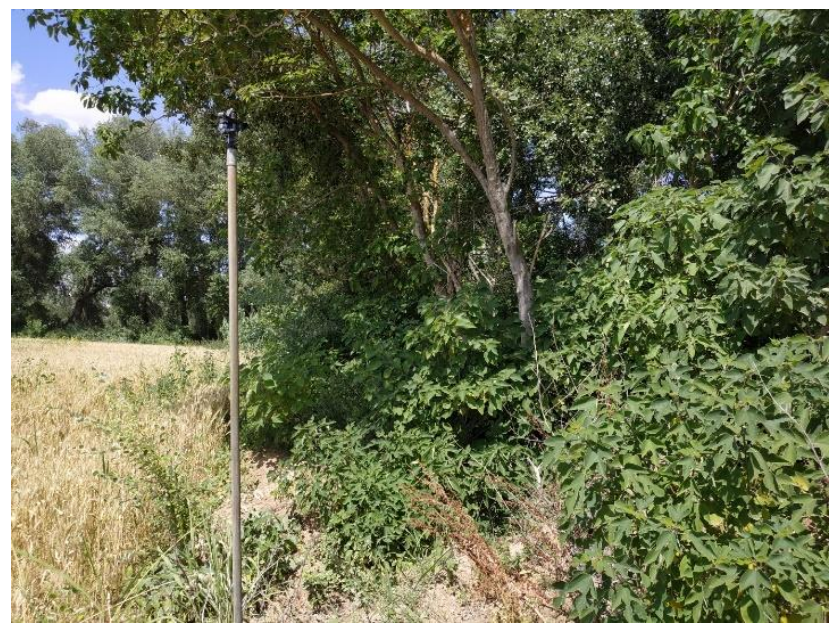

Figura 3. Imagen de la población de $B$. papyrifera detectada. Al fondo se aprecia una chopera de Populus alba.

Figure 3. Image of the population of $B$. papyrifera detected. In the background we can see a poplar tree of Populus alba.

Además de naturalizarse, B. papyrifera tiene un carácter invasor que está bien documentado. La base de datos Global Register of Introduced and Invasive Species (GRIIS, 2020) recoge evidencias de su impacto ambiental en Pakistán, donde se considera una de las seis peores plantas invasoras (Malik \& Hussain, 2007). En Argentina, donde se ha constatado que afecta a corredores y zonas riparias de la pampa (Ghersa et al. 2002). O en Ghana, donde desplaza a formaciones de vegetación autóctona (Bosu, et al. 2013). Además, esta base de datos también recoge incidencias en países como Bulgaria, Croacia, Uganda o Tanzania. En el conjunto de Europa, según la European and Mediterranean Plant Protection Organization, está incluida en el listado de observación de plantas exóticas invasoras (EPPO, 2020).

En España, B. papyrifera no se reconoce como una de las principales plantas invasoras (SanzElorza et al. 2004). Aunque, otros autores, en enclaves como el Parque Natural de Montseny (Barcelona), sí la han considerado como tal (Salvat \& Ernériz, 2017). Bayón \& Vilà (2019), la incluyen en un listado de atención, donde se recogen todas las especies invasoras y potencialmente invasoras de España con impactos ambientales o impactos socioeconómicos potenciales. Por tanto, el papel de B. papyrifera como planta invasora en España debe vigilarse con atención. 


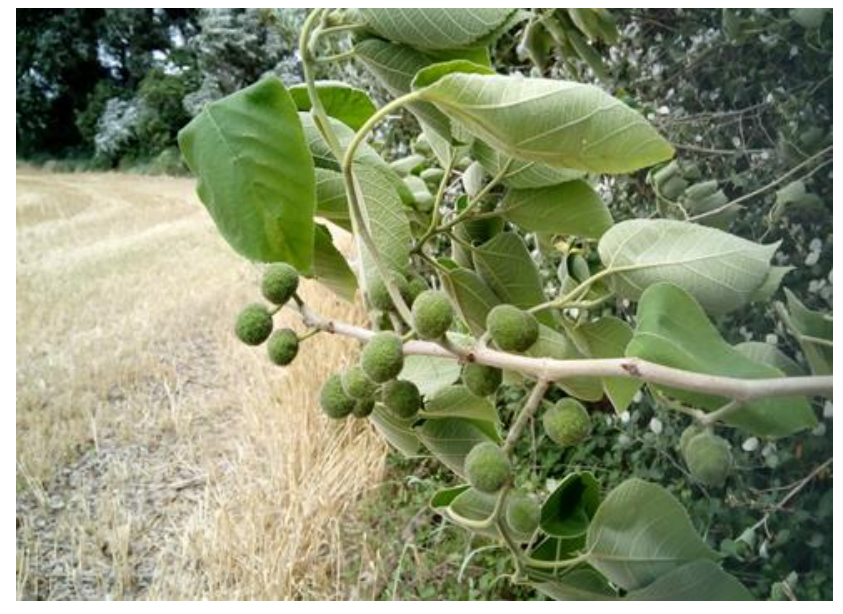

Figura 4. Detalle de los glomérulos femeninos de uno de los ejemplares.

Figure 4. Detail of the female glomeruli of one specimen.

La biología reproductiva de la especie favorece su potencial colonizador. La base de datos Invasive Species Compendium (CABI, 2020) indica que las aves pueden ser su agente dispersante, y alerta de que su eliminación es altamente difícil tras su introducción. En la costa NE española, se ha citado que su principal método de propagación es por semillas (Sobrino et al. 2002). Sin embargo, también parece preocupante su capacidad de propagación vegetativa, pues se regenera fácilmente a partir de los tallos cortados y los chupones de las raíces, que tienen un rápido crecimiento (EPPO, 2020). A esto, hay que sumar que se adapta a una amplia variedad de suelos y condiciones climáticas, lo que vendría a reforzar aún más su potencial invasor.

\section{Xenotipo y problemática de la población detectada}

Atendiendo a las categorías propuestas por Kornás (1990), sería un metáfito kenófito holoagriófito. Es decir, ejemplares asentados de modo permanente y en fecha relativamente reciente en ambientes naturales (como la orilla y vega del río). Esto contrastaría con el criterio de Sanz-Elorza, et al. (2004), que la consideran un metafito epecófito (que se establece en ambientes ruderales 0 arvenses). Por tanto, el presente trabajo refuerza la idea de que, en el centro peninsular, la especie también puede ocupar ambientes naturales y no solo antropizados.

La población hallada ocupa un rodal de la margen izquierda del río de unos $4.000 \mathrm{~m}^{2}$. Su presencia debe considerarse preocupante, ya que se encontraron numerosas plantas adultas (todas ellas vigorosas) y también rebrotes con nuevos ejemplares.

Se desconoce cómo se introdujo en el lugar, aunque la proximidad de una casa con jardín en la que existen ejemplares de la especie, hace pensar que escaparon de ahí hasta llegar a la ubicación detectada.
En Castilla-La Mancha, no se han encontrado referencias previas que hagan pensar que existen otras poblaciones naturalizadas en riberas. Por el contrario, las referencias consultadas en GBIF (2020) para el centro peninsular, suelen ubicarlas en zonas próximas a núcleos de población, carreteras, etc.

En la ribera indicada existe un bosquete autóctono de Populus alba L. que parece estar siendo desplazado por esta población emergente de $B$. papyrifera. La capacidad de esta especie para remplazar a la vegetación riparia ha sido puesta de manifiesto por Costa et al. (1986) en Valencia, donde denunciaron que "localmente se hace dominante por su acusado carácter colonizador" desplazando a olmedas hipernitrófilas. Esto, unido a que el río puede transportar aguas abajo sus propágulos, da una idea del riesgo de expansión de la especie.

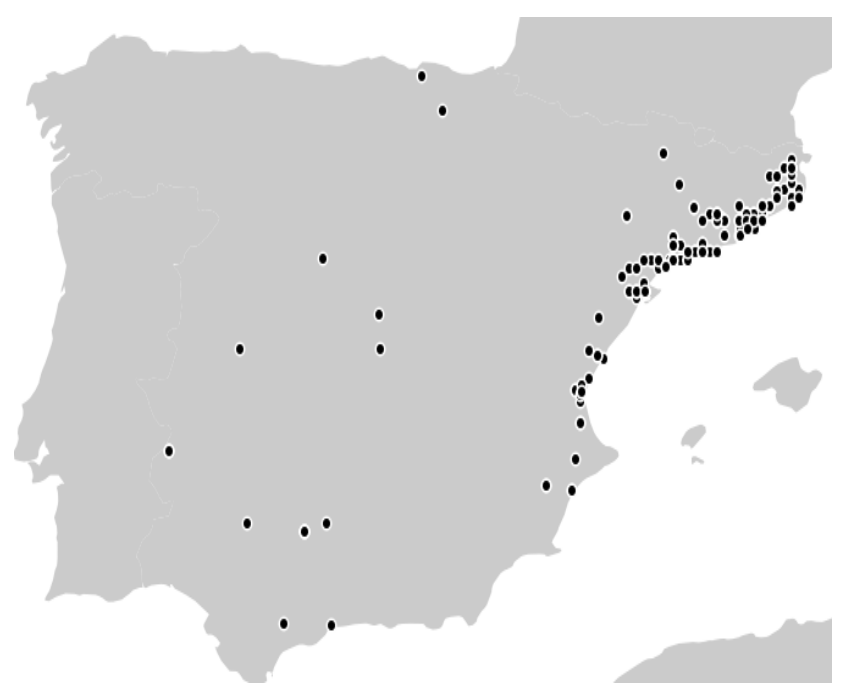

Figura 5. Distribución de Broussonetia papyrifera en la Península Ibérica. Fuente Global Biodiversity Information Facility (consultado en Junio de 2020).

Figure 5. Distribution of Broussonetia papyrifera in the Iberian Peninsula. Source Global Biodiversity Information Facility (accessed June 2020).

Por tanto, no es descartable que en el futuro aparezcan nuevas poblaciones. Como agravante, habría que valorar que río abajo existen varios espacios de la Red Natura que podrían verse afectados. Entre ellos, los más cercanos pertenecen a la Comunidad de Madrid y son la ZEPA ES0000119 (Carrizales y sotos de Aranjuez) y el ZEC ES31100006 (Vegas, Cuestas y Páramos del Sureste de Madrid) ambos a unos $10 \mathrm{Km}$.

De este modo, para evitar la expansión de la especie y los efectos negativos que ello pudiera acarrear, es necesario el control y vigilancia de la población encontrada. Por esta razón, se hace hincapié en la importancia de que las distintas administraciones con competencias tengan constancia de la presencia de $B$. papyrifera en las riberas del Tajo y se tomen las medidas oportunas. 


\section{Bibliografía}

Bayón, Á., \& Vilà, M. (2019). Horizon scanning to identify invasion risk of ornamental plants markete in Spain. NeoBiota, 52, 47-86 doi: https://doi.org//10.3897/neobiota.52.38113

Bolòs, O. de \& Vigo, J. (1990). Flora dels Països Catalans. vol. 2: (Crucíferes-Amarantàcies). Barcelona

Bosu, P., Apetorgbor, M. M., E. Nkrumah, Elvis \& P. Bandoh, Kwabena. (2013). The impact of Broussonetia papyrifera (L.) Vent. on community characteristics in the forest and forest-savannah transition ecosystems of Ghana. African Journal of Ecology. 51(4), 528-535 https://doi. org/10.1111/aje. 12063

CABI 2020. Broussonetia papyrifera In Invasive Species Compendium. Wallingford, UK: CAB International.

https://www.cabi.org/isc/datasheet/10017 [consultado 17 Junio 2020]

Castroviejo, S. (1993). Moraceae In Castroviejo, S., Aedo, C., Laínz, M., Muñoz Garmendia, F., Nieto Feliner, G., Paiva, J. \& Benedí, C. (Eds.), Flora iberica 3, 251-256. Real Jardín Botánico, CSIC, Madrid.

Costa, M., Peris, J. B., \& Stübing, G. (1986). Notas corológicas levantinas VI. Studia Botanica, 5, 123-125.

De Almeida \& Freitas. (2006). Exotic flora of Continental Portugal-A reassessment. Botanica complutensis, 30, 117-130.

EPPO (2020). European and Mediterranean Plant Protection Organization Global Database. https://gd.eppo.int/taxon/BRNPA/categorization [consultado 17 Junio 2020]

GBIF (2020). Broussonetia papyrifera in Occurrence dataset https://www.gbif. org/species/5361944 accessed via GBIF. org on 2020-06-17

Ghersa, C. M., de La Fuente, E., Suarez, S., \& Leon, R. J. C. (2002). Woody species in the Rolling Pampa grasslands, Argentina. Agriculture, Ecosystems and Environment, 88, 271-278.

GRIIS (2020). Global Register of Introduced and Invasive Species. Accessed via http://www. griis. org/ on 2020-06-17

Guillot Ortiz, D. (2009). Flora ornamental española: aspectos históricos y principales especies. Monografías de la Revista Bouteloua, 8, 1-273.

Kornás, J. (1990). Plant invasions in Central Europe: Historical and ecological aspects. In Di Castri, F., Hansen, A. J. \& Debussche, M. (Eds.), Biological Invasions in Europe and the Mediterranean Basin, (pp. 19-36). Kluwer Acad. Publ. Dordrecht. The Netherlands.

Laguna, M. (1870). Resumen de los trabajos verificados por la Comisión de la Flora Forestal Española durante los años de 1867 y 1868. Madrid Impr. del Colegio Nacional de Sordomudos y de Ciegos.

Malik, R. \& Husain, S. Z. (2007). Broussonetia payprifera: an environmental constraint on the Himalayan foothills vegetation. Pakistan Journal of Botany, 39, 1045-1053.

Salvat, A \& Ernériz J (2017). Parc Natural del Montseny: Plantes exòtiques invasores: Guia d'identificació i substitució en jardineria. Diputación de Barcelona.

Sanz-Elorza, M., Dana, E. D. \& Sobrino, E. (2004, eds.). Atlas de las Plantas Alóctonas Invasoras en España. Dirección General para la Biodiversidad. Madrid.

Sobrino, E., Sanz-Elorza, M., Dana, E. D. \& González-Moreno, A. (2002). Invasibility of a coastal strip in NE Spain by alien plants. Journal of Vegetation Science, 13(4), 585-594. 